Article

\title{
Optimal Energy Management for Hybrid Electric Vehicles Based on Dynamic Programming and Receding Horizon
}

\author{
Pierpaolo Polverino $^{1, *(\mathbb{D})}$, Ivan Arsie ${ }^{2}$ and Cesare Pianese ${ }^{1}$ (D) \\ 1 Department of Industrial Engineering, University of Salerno, Via Giovanni Paolo II 132, \\ 84084 Fisciano, SA, Italy; pianese@unisa.it \\ 2 Department of Engineering, University of Naples "Parthenope", Centro Direzionale, \\ 80143 Napoli, NA, Italy; ivan.arsie@uniparthenope.it \\ * Correspondence: ppolverino@unisa.it; Tel.: +39-089-96-4178
}

Citation: Polverino, P.; Arsie, I.;

Pianese, C. Optimal Energy

Management for Hybrid Electric Vehicles Based on Dynamic

Programming and Receding Horizon.

Energies 2021, 14, 3502. https://

doi.org/10.3390/en14123502

Academic Editor: Felix Barreras

Received: 29 March 2021

Accepted: 8 June 2021

Published: 12 June 2021

Publisher's Note: MDPI stays neutral with regard to jurisdictional claims in published maps and institutional affiliations.

\begin{abstract}
Fuel consumption and emissions in parallel hybrid electric vehicles (HEVs) are directly linked to the way the load request to the wheels is managed between the internal combustion engine and the electric motor powered by the battery. A significant reduction in both consumption and emissions can be achieved by optimally controlling the power split on an entire driving mission (full horizon-FH). However, the entire driving path is often not predictable in real applications, hindering the fulfillment of the advantages gained through such an approach. An improvement can be achieved by exploiting more information available onboard, such as those derived from Advanced Driver Assistance Systems (ADAS) and vehicle connectivity (V2X). With this aim, the present work presents the design and verification, in a simulated environment, of an optimized controller for HEVs energy management, based on dynamic programming (DP) and receding horizon (RH) approaches. The control algorithm entails the partial knowledge of the driving mission, and its performance is assessed by evaluating fuel consumption related to a Worldwide harmonized Light vehicles Test Cycle (WLTC) under different control features (i.e., horizon length and update distance). The obtained results show a fuel consumption reduction comparable to that of the $\mathrm{FH}$, with maximum drift from optimal consumption of less than $10 \%$.
\end{abstract}

Keywords: electrified powertrain; energy management strategy; look-ahead control; hybrid vehicles; dynamic programming; optimal control; receding horizon

\section{Introduction}

The widespread use of fossil fuels in the automotive field has been considered in the last few decades as one of the major contributors responsible for urban pollution and climate change. These issues have created a strong impulse towards the development of alternative propulsion systems that could guarantee competitive features vs. conventional internal combustion engine (ICE)-based powertrains in terms of operational costs, performance, and autonomy, as well as industrial feasibility. In this scenario, hybrid-electric powertrains have proven to be a good solution as they allow the performance of a smooth transition from ICE-based to full-electric powertrains, thus allowing the benefits of both solutions. Indeed, HEVs allow the achieving of low exhaust and acoustic emissions, significant energy savings, and improved drivability, typical of an electric powertrain, along with the high autonomy guaranteed by ICE [1,2].

The key point for the proper operation of HEVs is the energy management that is aimed at defining the most suitable power split between the multi-source energy systems available onboard. The energy management strategy (EMS) is in charge of instantaneously evaluating the optimal power ratio between ICE and electric motor-generator (EMG), in relation to the requested drive power and the available energy in the battery (i.e., its stateof-charge-SoC), with the aim of minimizing fuel consumption and exhaust emissions. 
Recently, a remarkable amount of research studies have been carried out on EMS, with different methodologies and goals, depending on HEV topology (e.g., series, parallel, plug-in), mathematical formulation (e.g., rule-based, optimization-based) and available route information (e.g., causal, non-causal) [3,4]. Particularly, EMSs based on optimal control theory (i.e., optimization-based) allow the achieving of a global optimum solution, but they require the a-priori knowledge of vehicle velocity trajectory (i.e., non-causal). In this framework, dynamic programming (DP) and Pontryagin's minimum principle (PMP) are widely used in the literature $[5,6]$ for vehicle optimization problems $[7,8]$. On the other hand, in the majority of the real-world driving applications, a vehicle's velocity trajectory is not available since it is determined by the causal interaction of the vehicle with the environment and other vehicles along the route. Therefore, the aforementioned techniques (i.e., DP and PMP) are implemented in offline global optimization research to achieve benchmark solutions for supporting the onboard EMS design or the powertrain system configuration/sizing [9]. Nevertheless, other optimization-based techniques, such as equivalent consumption minimization strategy (ECMS) and model predictive control (MPC), are extensively used for real-time EMS, but only a sub-optimal solution can be achieved in these cases [10-12].

In order to overcome these issues, a speed prediction scheme has to be implemented in the supervisory controller, which allows evaluating the globally optimized EMS along the estimated load trajectory. The prediction can be performed by making use of both historical data and look-ahead information, retrieved by onboard vehicle sensors and connectivity [13]. Hedge et al. [14] presented a methodology to predict the vehicle's speed trajectories by look-ahead data and concluded that good accuracy could be achieved by suitably tuning the level of look-ahead data with the route typology. The authors proposed in [15] a load estimate via a recurrent neural network coupled with DP to optimize the supervisory control strategy during a future time horizon.

In this context, the purpose of this work is to design a supervisory controller for the optimal EMS of a parallel HEV based on the application of DP over a receding horizon (RH) of limited length derived from ADAS and V2X technologies. The proposed methodology allows limiting the uncertainty of predicting the whole speed trajectory and makes the optimal control solution more realistic.

\section{Hybrid Electric Vehicle (HEV) Model}

The HEV architecture accounted for here is a parallel one, and it is depicted in Figure 1. The power requested at the wheels is supplied by an ICE and an EMG powered by a battery. The energy losses are mainly related to the mechanical transmission from the engine and the motor to the wheels due to the presence of a clutch, a gearbox, a coupler and a differential, and also to the electric transmission between the battery and the motor through the power link.

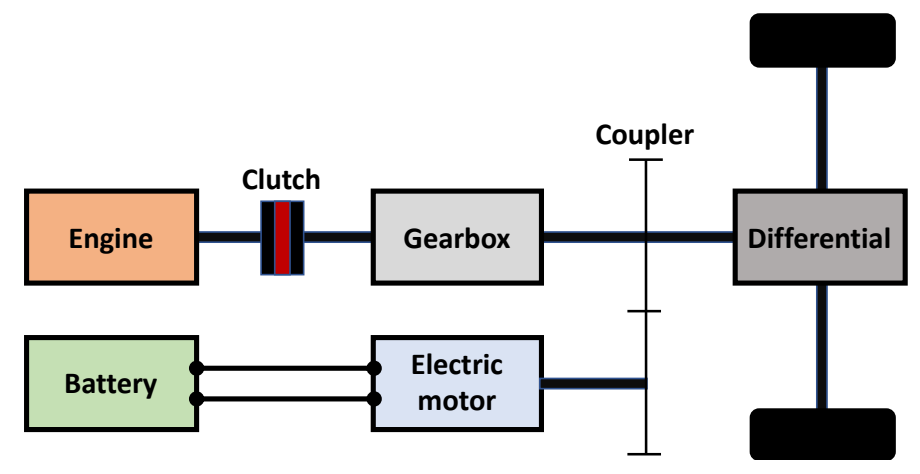

Figure 1. Schematic representation of the considered parallel hybrid electric vehicle (HEV) architecture. 
To evaluate the power requested at the wheels, the optimal vehicle speed is accounted for given a certain driving path, and the wheel's power request is computed through a vehicle longitudinal dynamics model.

\subsection{Vehicle Longitudinal Dynamics Model}

The equation that describes the longitudinal dynamics of the vehicle is expressed in the following $[2,12]$ :

$$
m_{v} \frac{d v}{d t}=F_{w}-F_{d}-F_{s}-F_{r}
$$

where $m_{v}$ is the overall vehicle mass, $F_{w}$ is the driving force at the wheels, $F_{d}$ is the aerodynamic drag force, $F_{s}$ is the gravity force component that affects vehicle motion due to road slope, and $F_{r}$ is the rolling friction force:

$$
\begin{gathered}
F_{d}=\frac{1}{2} c_{d} \rho_{a i r} A v^{2} \\
F_{s}=m_{v} g \sin \alpha \\
F_{r}=m_{v} c_{r} g \cos \alpha
\end{gathered}
$$

Given a certain driving path, upon the knowledge of the vehicle mass $m_{v}$, desired speed $v$, and road slope $\alpha$, Equation (1) can be rearranged to evaluate the driving force at the wheels [2]:

$$
F_{w}=m_{v} \frac{d v}{d t}+F_{d}+F_{s}+F_{r}
$$

which can be then used to estimate the driving torque $T_{w}$, rotational speed $\omega_{w}$ and overall driving power $P_{w}$.

\subsection{Transmission Model}

The transmission model refers to the whole mechanical transmission chain depicted in Figure 1. The power generated by the engine is transferred to the wheels through the clutch $(c l)$, gearbox $(g)$ and differential $(d)$. The whole transmission efficiency and ratio are:

$$
\begin{gathered}
\eta_{e 2 w}^{G}=\eta_{c l} \eta_{g}^{G} \eta_{d} \\
\gamma_{e 2 w}^{G}=\gamma_{g}^{G} \gamma_{d}
\end{gathered}
$$

Both gearbox efficiency and ratio depend on the applied gear $(G)$. Equation (7) leads to the relation between engine angular speed and that at the wheels:

$$
\omega_{e}=\omega_{w} \gamma_{e 2 w}^{G}
$$

Similarly, the power generated by the electric motor is transferred to the wheels thanks to the coupler $(c p)$, accounting for the following transmission efficiency and ratio:

$$
\begin{aligned}
\eta_{m 2 w} & =\eta_{c p} \eta_{d} \\
\gamma_{m 2 w} & =\gamma_{c p} \gamma_{d}
\end{aligned}
$$

Furthermore, the angular speed of the electric motor is linked to that at the wheels as:

$$
\omega_{m}=\omega_{w} \gamma_{m 2 w}
$$

Battery recharging can be achieved by taking power from the engine (charging) or by recovering power from the wheels during deceleration (regenerative braking). The charging process is characterized by the following efficiency:

$$
\eta_{e 2 m}^{G}=\eta_{c l} \eta_{g}^{G} \eta_{c p}
$$


whereas regenerative braking efficiency is the same as that reported in Equation (9).

\subsection{Engine and Electric Motor Models}

The engine type accounted for in this work is a port fuel-injection spark-ignition (SI) engine, with a displacement of $1.2 \mathrm{~L}$, four cylinders in-line and a max torque of $105 \mathrm{Nm}$ at $3500 \mathrm{rpm}$. The engine is modeled considering a map-based approach to describe the relations among the engine brake torque efficiency, speed and torque, as depicted in Figure 2a.
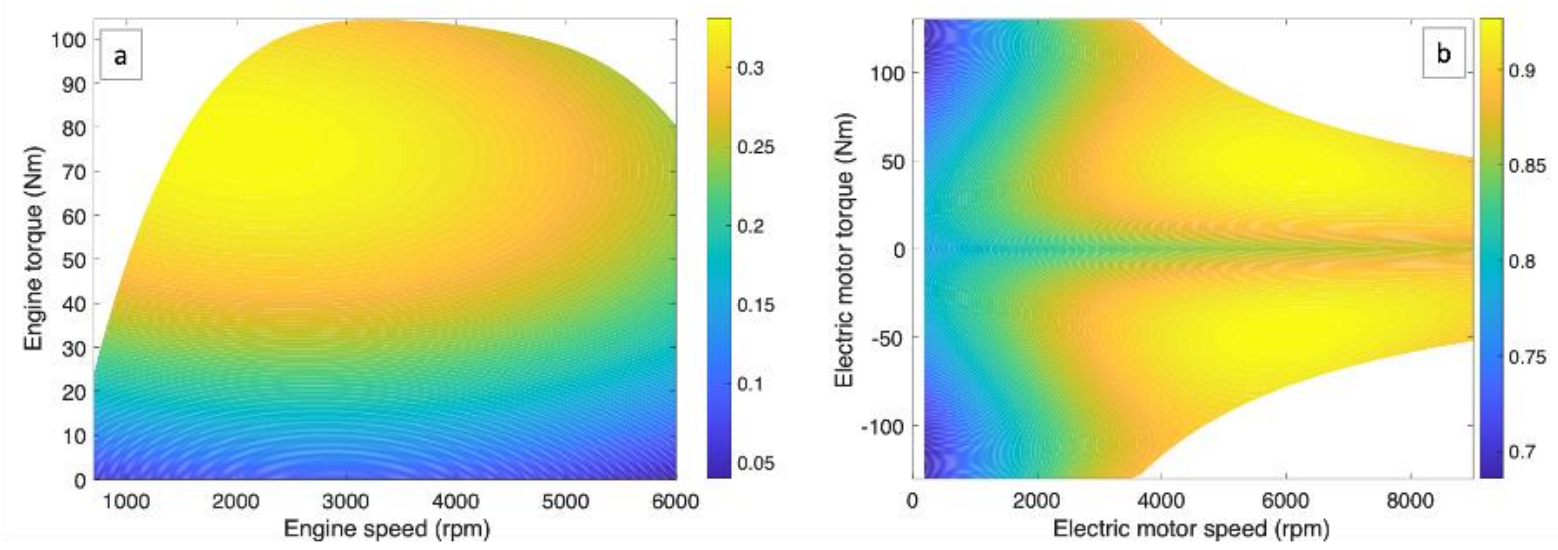

Figure 2. SI engine (a) and electric motor (b) efficiency maps as a function of speed and torque.

The electric motor is modeled as a permanent magnet synchronous reversible machine that converts electric power from the battery into mechanical power (available at the wheels) during traction mode, and vice-versa in battery recharging mode (taking mechanical power from the wheels during regenerative braking or from the engine during charging).

Considering the same map-based approach used for the engine, the motor efficiency is represented through the map in Figure 2b, where two main regions can be identified: (i) a low-speed region with constant drive torque and (ii) a high-speed region with constant drive power. The maximum speed and torque are $9000 \mathrm{rpm}$ and $130 \mathrm{Nm}$, respectively, and the transition speed (from low-speed to high-speed regions) is about $3200 \mathrm{rpm}$.

\subsection{Battery Model}

The battery pack is made of 30 parallel branches of 24 cells in series, each with a maximum capacity of $33 \mathrm{Ah}$ and an overall mass of $30 \mathrm{~kg}$. The single cells are modeled accounting for an equivalent circuit representation [12], in which the output voltage is related to the ideal voltage through the internal resistance as follows:

$$
V_{b}^{\text {cell }}=V_{i d}^{\text {cell }}-I_{b}^{\text {cell }} R_{b}^{\text {cell }}
$$

where the term $I_{b}^{\text {cell }}$ represents the current flowing through the battery. The battery state of charge (SOC) can be then expressed as the ratio between the current battery charge and the maximum one:

$$
\xi=\frac{Q(t)}{Q_{\max }}
$$

and its change over time is expressed as:

$$
\frac{d \xi}{d t}=\frac{I_{b}^{\text {cell }}}{Q_{\max }}
$$

It is worth recalling that the internal resistance expressed in Equation (13) changes according to the battery operation, i.e., charging or discharging mode. 


\section{Receding Horizon (RH)-Based Energy Management Strategy (EMS)}

A parallel hybrid architecture has one degree of freedom through which power flows can be managed optimally, considering the different operating modes. Therefore, as remarked in the introduction section, fuel consumption can be minimized by managing these energy flows with a suitable energy management strategy (EMS) that involves a supervisory controller $[9,16]$.

The strategies that can be adopted by the supervisory controller can be divided into two main classes: optimal and heuristic. The former uses optimal control theory to formulate a problem that can be solved subsequently, whereas the latter follows rulebased indications calibrated in advance through optimal control techniques. Another classification can be performed according to the used information: causal and non-causal. Non-causal control strategies require the full knowledge of all inputs (e.g., those related to the travel path). On the other hand, causal strategies use only past and present information.

Energy management optimization could be performed either offline or online: offline design generally exploits the knowledge of the driving cycle in advance. Nevertheless, when the driving cycle changes during operation, online approaches are required. Offline optimization generally uses a non-causal strategy (i.e., complete knowledge of the entire future path) to achieve minimum fuel consumption [4]. Conversely, online optimization uses causal control strategies (i.e., only knowledge of the previous and current state), but they can also use non-causal control strategies when the vehicle follows a predetermined path (e.g., public transports, trains) or when future driving conditions are somehow predicted [11].

The formulation of the basic optimization problem consists of the choice of a control variable $u(t)$ to maximize (or minimize) an integral objective function $J$ :

$$
J(u(t))=\int_{0}^{T} L(x(t), u(t), t) d t
$$

where $x(t)$ represents the state variable and $L$ is an instantaneous cost function that combines several pieces of information defined for the specific problem. In HEVs, the state variable that is usually considered is the battery SOC. When accounting for a charge sustaining approach, to consider the constraint on the final SOC, the objective function is formulated adding a penalty function:

$$
J(u(t))=\int_{0}^{T} L(x(t), u(t), t) d t+\phi(\xi(T))
$$

Among the different procedures to solve an optimal control problem, dynamic programming (DP) allows the finding of the optimal global solution respecting multiple constraints. The main disadvantage is its heavy computational load, which increases exponentially with the number of system state and control variables [12]. DP was firstly introduced in 1957 as a solution for multi-stage decision problems in which time plays a significant role, and the order of operations may be crucial [17].

The specific problem objective of this study can be presented as the identification of the optimal power profile (or the optimal power split among the battery and thermal engine) to be applied according to the mission profile in order to minimize the overall fuel consumption of the vehicle. Therefore, the instantaneous cost function becomes the fuel mass flow, whereas the system state and control variables are the battery SOC and power, respectively:

$$
J(u(t))=\int_{0}^{T} \dot{m}_{f}\left(\xi(t), P_{b}(t), t\right) d t+\phi(\xi(T))
$$

The minimum and maximum battery power values are strictly related to the battery charge and discharge features. The engaged gear is chosen according to the one that gives 
the lowest fuel consumption, whereas the minimum and maximum limits for the battery SOC are imposed and represent an input for the strategy implementation.

DP implementation requires the discretization of time as well as state and control variable feasible sets. Thus, the solution found with the DP can be considered optimal within the error limits introduced by the discretization. The overall mission cost in Equation (18) can be written as:

$$
J=\sum_{k=0}^{N-1} \dot{m}_{f}\left(t_{k}, P_{b}\left(t_{k}\right)\right)+\phi\left(\xi\left(t_{N}\right)\right)
$$

where integral form is now substituted by the summation of the costs related to the $N$ time intervals and the final cost, the DP algorithm starts from the end of the mission profile and proceeds backward in time towards the start of the mission. During this process, at each $k$-th discrete time instant, the algorithm stores the optimal value of the battery power and the cost-to-go $\left(J_{k}\right)$ that represents the minimum cost related to the transition from each $i$-th SOC at $t_{k}$ to the SOC at the end $\left(t_{N}\right)$. After computing all the costs for the final SOC to all possible initial SOC values, the algorithm evaluates the optimal state trajectory through a forward evaluation in time. More details on the DP approach can be found in [18,19].

In this work, the combination of the DP method with the receding horizon (RH) approach to find sub-optimal solutions with respect to those achieved with the knowledge of the entire driving path (full horizon-FH) is presented. In real applications, it is generally not possible to know the whole driving horizon, especially for long journeys, due to factors such as alternative routes, traffic. Therefore, if the optimal solution is calculated on a predetermined path, when, for some reason, the drivability conditions vary, the vehicle does not follow the optimal trajectory programmed but a non-optimal one. The RH approach allows the solving of the problem of optimal control on a shorter horizon and to update the solution with a periodic interval (which could be either in time or in space) $[13,14]$. Hence, two main parameters need to the defined as illustrated in the example of Figure 3: (i) the path length $\left(H_{\mathrm{p}}\right)$ and (ii) the updating interval $\left(H_{\mathrm{s}}\right)$. It is clear that the choice of such parameters is fundamental to achieve a satisfactory solution that represents a compromise between the optimal solution achieved with the FH and the practical applicability of the RH-based approach.

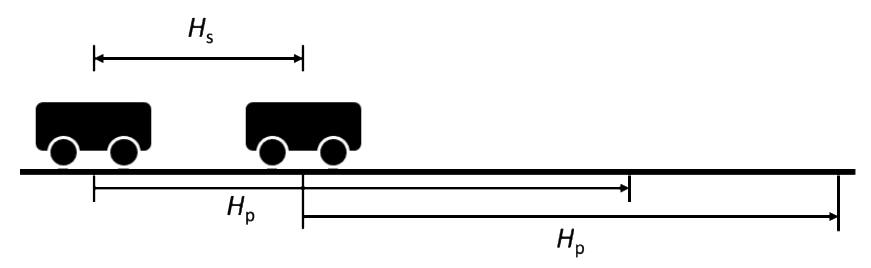

Figure 3. Receding Horizon approach with identification of the path length $H_{\mathrm{p}}$ and updating interval $H_{\mathrm{s}}$.

\section{Case Study Results and Discussion}

The RH-based EMS is applied to a WLTC driving cycle, and the performance in terms of fuel consumption is investigated with respect to that achieved with an $\mathrm{FH}$ approach. Moreover, a parametric analysis is performed by varying $H_{\mathrm{p}}$ and $H_{\mathrm{s}}$ and observing the deviation of the fuel consumption so as to identify the limit conditions under which the EMS solution degrades with respect to the FH solution.

Figure $4 \mathrm{a}, \mathrm{b}$ presents the desired vehicle speed and the related traveled distance, respectively, for the WLTC cycle, whereas Figure 4c represents the slope accounted for in the study and Figure $4 \mathrm{~d}$ reports the traveled height related to the applied slope and desired speed. For the initial urban path, an increasing slope is assumed, followed by a decrease and a stabilization in the last highway section of the cycle. As previously commented, the battery management accounts for a charge sustaining procedure, with minimum and maximum battery SOC being 0.5 and 0.7 , respectively. Moreover, the initial SOC is assumed to be 0.6 , which needs to be respected at the end of the whole FH optimization as well as at the end of each $H_{\mathrm{p}}$ horizon for the RH optimization. 

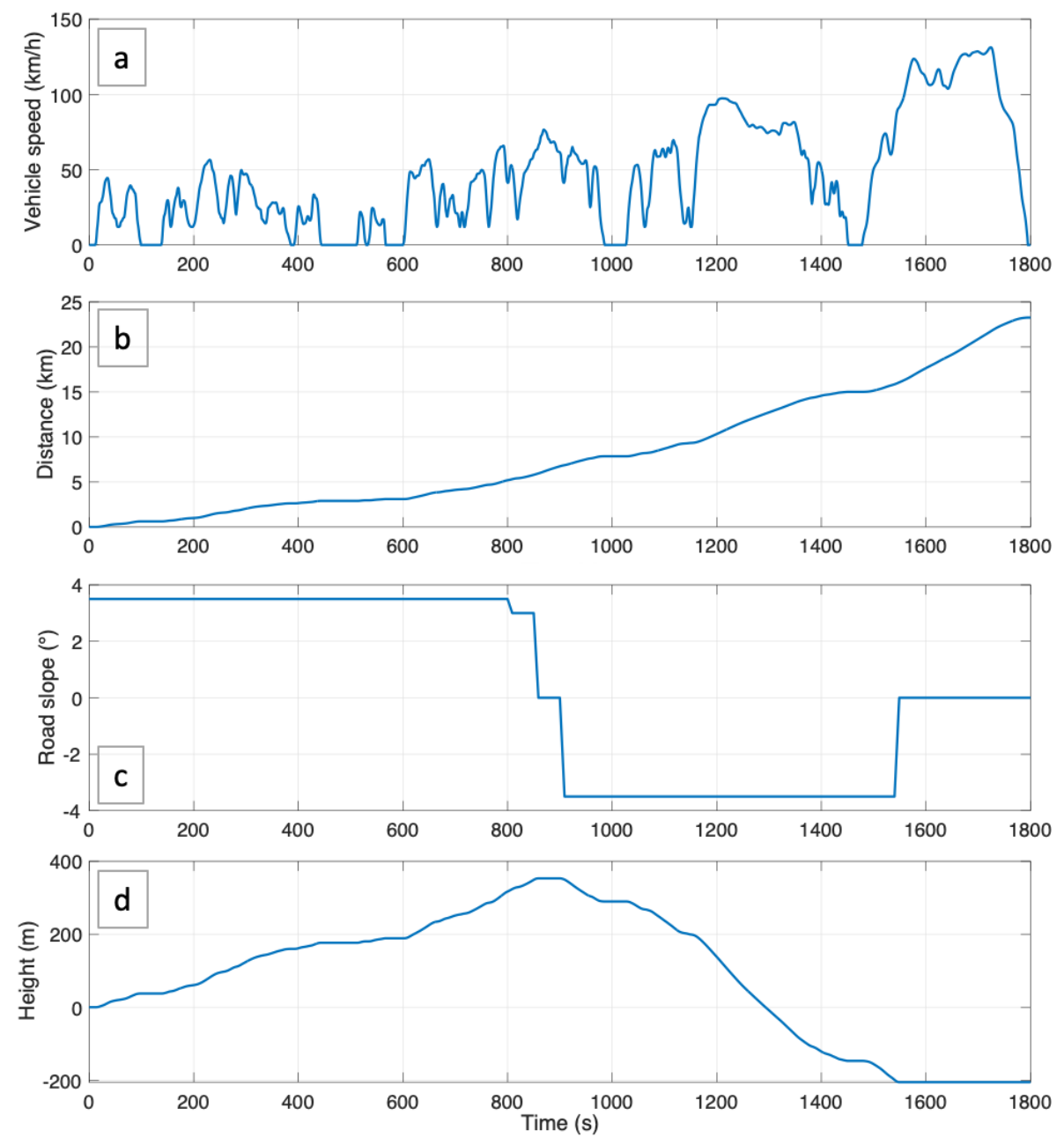

Figure 4. Desired speed (a), traveled distance (b), considered road slope (c) and related traveled height (d) for the accounted WLTC cycle.

Figure 5 reports with solid blue lines the results achieved with an $\mathrm{FH}$ approach on the considered cycle in terms of SOC (Figure 5a) and fuel consumption (Figure $5 b$ ) behaviors over time. It can be observed that the strategy based on the knowledge of the entire path asks for an almost continuous discharge of the battery until the minimum SOC is reached at $900 \mathrm{~s}$. Afterward, the battery is charged, taking advantage of both engine charging and regenerative braking, until the maximum allowed SOC is reached at around $1500 \mathrm{~s}$. The last part of the highway route accounts for battery discharge to respect the imposed SOC at the end of the path (i.e., 0.6 reached at $1800 \mathrm{~s}$ ).

Fuel consumption behavior over time reflects the constraints imposed by the cycle, especially those related to the slope. Although discharging the battery in the first part of the cycle, the fuel consumption increases with an almost constant slope. This is due to the need for traction due to the increase in road slope. Since the EMS is aware of the future road slope variation, the battery is used to overcome the initial part of the cycle where more energy is required, while in the second part, battery SOC can be recovered thanks to regenerative braking due to the reduction in path slope. This is indeed evident between $900 \mathrm{~s}$ and $1500 \mathrm{~s}$, where the fuel consumption is almost constant at $0.6 \mathrm{~kg}$. The final part of the cycle sees a greater increase in fuel consumption instead due to the end of road slope change and to the higher speed requested. The final result is then $0.98992 \mathrm{~kg}$ of fuel consumed for the entire cycle. 

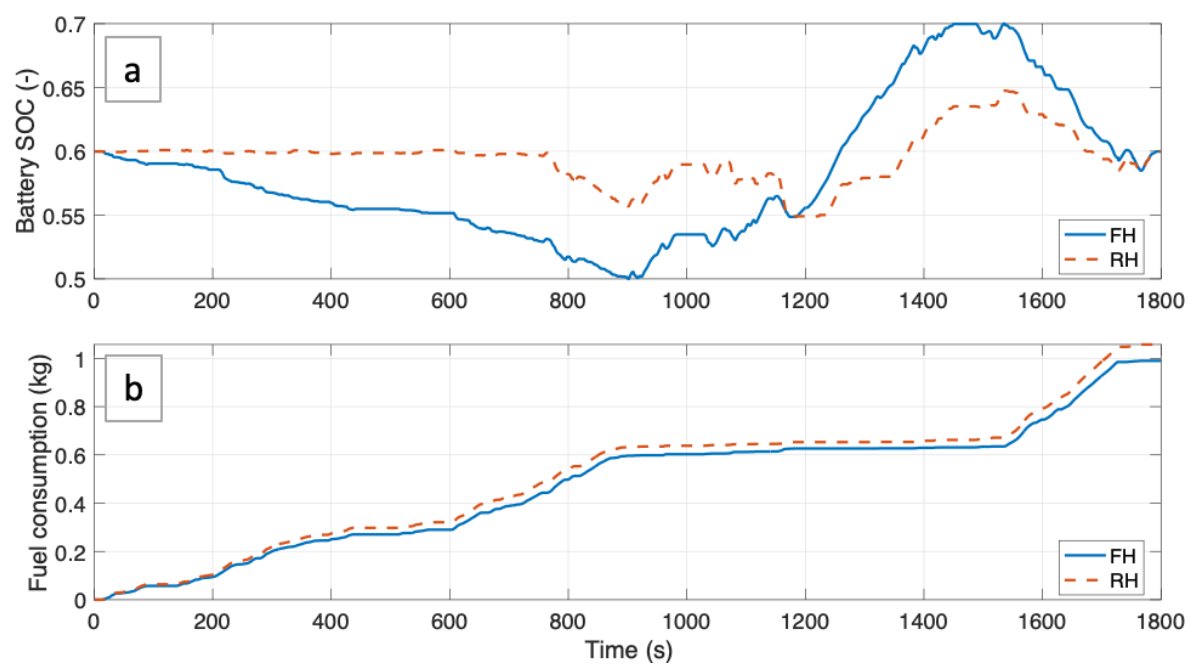

Figure 5. Comparison of battery optimal SOC time behavior (a) and fuel consumption trend (b) achieved with $\mathrm{RH}$ approach with a $H_{\mathrm{p}}$ window of $300 \mathrm{~s}$ and a $H_{\mathrm{s}}$ window of $150 \mathrm{~s}$ (red dashed lines) with respect to those achieved with the FH approach (solid blue lines) applied to a WLTC cycle (see Figure 4).

The application of the RH-based approach requires the setting of both the partial horizon $H_{\mathrm{p}}$ and the update interval $H_{\mathrm{s}}$. For a preliminary analysis, an initial value of $300 \mathrm{~s}$ is chosen for the $H_{\mathrm{p}}$, whereas the $H_{\mathrm{s}}$ is set to $150 \mathrm{~s}$. This means that the WLTC cycle is analyzed with a sliding window of $300 \mathrm{~s}$ that is updated every $150 \mathrm{~s}$. The results of this process are compared to those achieved with the FH in Figure 5a in terms of SOC time behavior and in Figure $5 b$ in terms of fuel consumption with red dashed lines. From Figure 5a, it can be observed that, differently from the FH results, the SOC is practically kept constant at around 0.6 in the first part of the cycle. This is due to the fact that the algorithm can only update the path knowledge with a narrower window and, since the road slope increases up to $900 \mathrm{~s}$, the algorithm cannot consume battery energy without defying the constraint on the SOC at the end of the $H_{\mathrm{p}}$ window. This condition changes as soon as the algorithm encounters a slope inversion within the $H_{\mathrm{p}}$ window, as can be seen after $750 \mathrm{~s}$. This indeed allows the recharging of the battery after its use. The second part of the cycle has similar behavior, although smoothed, in terms of SOC trend to that of FH since the algorithm can use more of the battery (being in descent), and the recharging process takes place before the final part of the highway route to fulfill the charge sustaining constraint. The change in SOC behavior is reflected in a change in the final fuel consumption achieved by the RH approach. However, although the SOC trend is quite different, the fuel consumption trend is almost the same if compared to that of the FH approach, as can be seen in Figure $5 b$. As a final result, the fuel consumption achieved for the WLTC cycle with the imposed $H_{\mathrm{p}}$ and $H_{\mathrm{s}}$ windows is about $1.0574 \mathrm{~kg}$, with an increase from FC consumption of $6.8 \%$.

To investigate the change in EMS results with different $H_{\mathrm{p}}$ windows, the same $H_{\mathrm{s}}$ value of $150 \mathrm{~s}$ is considered, and two further values of $200 \mathrm{~s}$ and $400 \mathrm{~s}$ are accounted for $H_{\mathrm{p}}$. The related results in terms of SOC trend are shown in Figure 6 with respect to the $\mathrm{FH}$, and the $\mathrm{RH}$ results formerly discussed. It can be immediately seen that, by increasing the horizon (i.e., the path) length, the SOC trend tends towards that of the $\mathrm{FH}$, as obviously expected. 


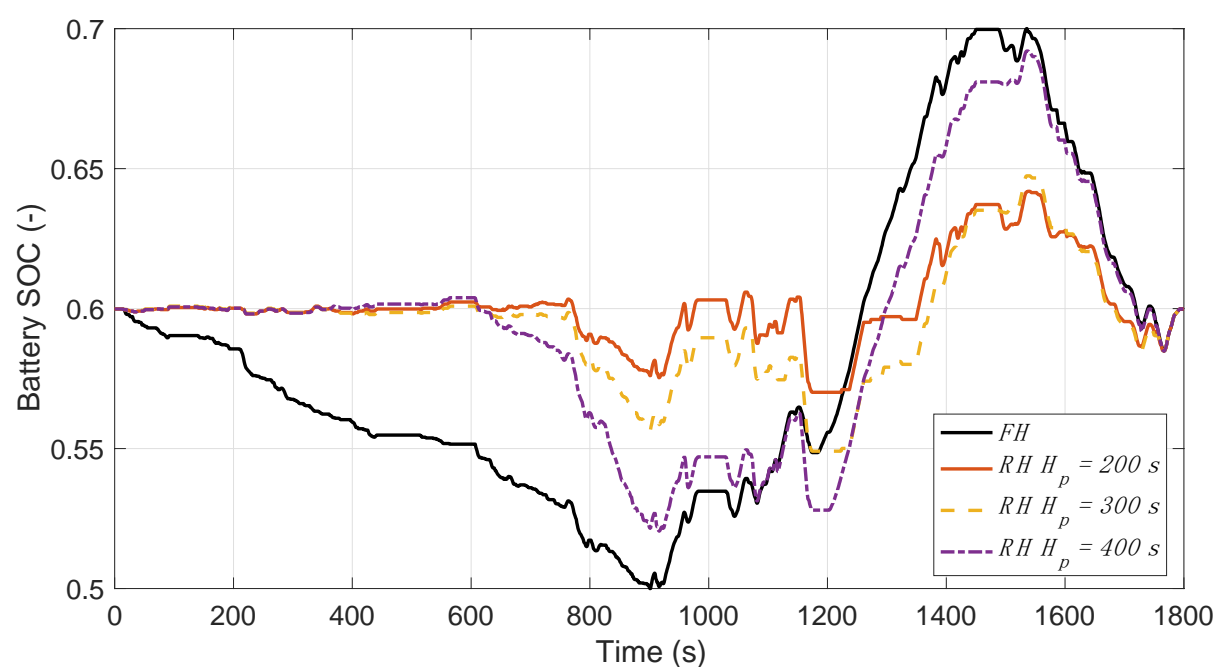

Figure 6. Comparison of SOC trends achieved for the RH approach with different $H_{\mathrm{p}}$ windows (constant $H_{\mathrm{s}}$ of $150 \mathrm{~s}$ ).

The achieved result in terms of fuel consumption for $H_{\mathrm{p}}=200 \mathrm{~s}$ is $1.0622 \mathrm{~kg}$, with an increase of $7.3 \%$ with respect to $\mathrm{FH}$ consumption, while for $H_{\mathrm{p}}=400 \mathrm{~s}$, the fuel consumption is $1.0081 \mathrm{~kg}$ (an increase of $1.83 \%$ ). The achieved results prove that with a $H_{\mathrm{p}}$ window above $400 \mathrm{~s}$, the results are almost comparable with those of the $\mathrm{FH}$, with a fuel consumption increase lower than $2 \%$.

Further extending the parametric analysis to the $H_{\mathrm{s}}$ window length, different combinations of $H_{\mathrm{s}}-H_{\mathrm{p}}$ values are investigated, and the related results are presented in an integrated way in Figure 7. The optimal fuel consumption achieved with the FH approach is depicted with a dashed black line at the bottom of the figure. On the x-axis, the values of the $H_{\mathrm{p}}$ windows are reported, from $100 \mathrm{~s}$ up to $400 \mathrm{~s}$, while the different curves are parametric with respect to $H_{\mathrm{s}}(50 \mathrm{~s}, 100 \mathrm{~s}, 150 \mathrm{~s}$ and $200 \mathrm{~s})$. Some numerical results are presented in Table 1.

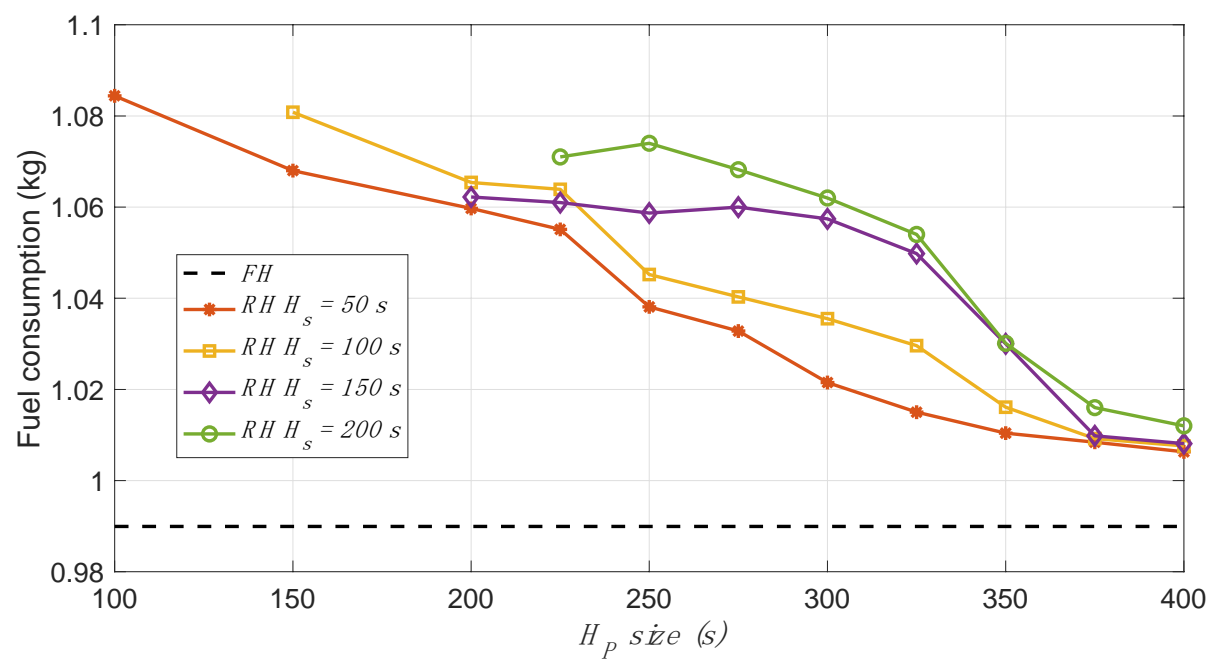

Figure 7. Fuel consumption trend with different $H_{\mathrm{p}}$ and $H_{\mathrm{s}}$ windows. 
Table 1. Fuel consumption results achieved with RH-based EMS compared with the FH approach with different $H_{\mathrm{s}}$ and $H_{\mathrm{p}}$.

\begin{tabular}{cccc}
\hline $\boldsymbol{H}_{\mathbf{s}}(\mathbf{s})$ & $\boldsymbol{H}_{\mathbf{p}}(\mathbf{s})$ & Fuel Consumption RH $\mathbf{( k g )}$ & Deviation from FH \\
\hline \multirow{3}{*}{50} & 100 & 1.0844 & $9.55 \%$ \\
& 200 & 1.0597 & $7.05 \%$ \\
& 300 & 1.0215 & $3.19 \%$ \\
& 400 & 1.0063 & $1.65 \%$ \\
\hline \multirow{2}{*}{100} & 200 & 1.0654 & $7.62 \%$ \\
& 300 & 1.0355 & $4.61 \%$ \\
\multirow{2}{*}{150} & 400 & 1.0076 & $1.79 \%$ \\
& 200 & 1.0622 & $7.30 \%$ \\
& 300 & 1.0574 & $6.82 \%$ \\
\multirow{2}{*}{200} & 400 & 1.0081 & $1.83 \%$ \\
& 300 & 1.0620 & $7.30 \%$ \\
\hline
\end{tabular}

From the trends reported in Figure 7 and the values illustrated in Table 1, it can be clearly assessed that fuel consumption decreases with an increasing path horizon $H_{p}$ while grows with the rise of the update length $H_{\mathrm{s}}$. The limit of $2 \%$ deviation from $\mathrm{FH}$ consumption is achieved with $H_{\mathrm{p}}$ values equal to $400 \mathrm{~s}$ and only if $H_{\mathrm{s}}$ is kept below $200 \mathrm{~s}$.

\section{Conclusions}

The optimal energy management of parallel HEVs based on the receding horizon approach has been addressed in this work. The optimization problem has been solved by combining a dynamic programming algorithm with a receding horizon $(\mathrm{RH})$ approach, imposing partial mission horizons (of length $H_{\mathrm{p}}$ ) and updating the solution at given time steps (update length $H_{\mathrm{s}}$ ). This approach allows overcoming the critical issue of predicting the whole driving mission (i.e., full horizon-FH) and enables the exploitation of ADAS and V2X technologies for the knowledge of the short-term mission profile. Furthermore, it is worth noting that solving an optimal control problem of reduced dimensionality allows reducing the computational demand, thus promoting the feasibility of real-time implementation. The proposed methodology has been analyzed in a simulation environment on a WLTC cycle with varying slope by evaluating the impact of $H_{\mathrm{s}}$ and $H_{\mathrm{p}}$ variations on the fuel consumption deviation vs. the FH solution, assumed as the benchmark. The results show that the fuel consumption decreases with the path horizon increase $\left(H_{\mathrm{p}}\right)$, while it increases with the growth of the update length $\left(H_{\mathrm{s}}\right)$. In particular, a deviation of lower than $2 \%$ from FH consumption is observed with $H_{\mathrm{p}}$ values equal to $400 \mathrm{~s}$ and $H_{\mathrm{s}}$ below $200 \mathrm{~s}$.

Author Contributions: Conceptualization, P.P., I.A. and C.P.; methodology, P.P. and I.A.; software, P.P.; validation, P.P.; formal analysis, P.P. and I.A.; investigation, P.P.; data curation, P.P. and I.A.; writing-original draft preparation, P.P.; writing-review and editing, P.P., I.A. and C.P.; supervision, C.P. All authors have read and agreed to the published version of the manuscript.

Funding: This research received no external funding.

Conflicts of Interest: The authors declare no conflict of interest.

\section{References}

1. Trana, D.; Vafaeipoura, M.; El Baghdadia, M.; Barrero, R.; Van Mierlo, J.; Hegazya, O. Thorough state-of-the-art analysis of electric and hybrid vehicle powertrains: Topologies and integrated energy management strategies. Renew. Sustain. Energy Rev. 2020, 119, 109596. [CrossRef]

2. Guzzella, L.; Sciarretta, A. Vehicle Propulsion Systems, Introduction to Modeling and Optimization; Springer: Berlin/Heidelberg, Germany, 2012.

3. Onori, S.; Serrao, L.; Rizzoni, G. Hybrid Electric Vehicles: Energy Management Strategies; Springer: Berlin/Heidelberg, Germany, 2016. 
4. Xue, Q.; Zhang, X.; Teng, T.; Zhang, J.; Feng, Z.; Lv, Q. A Comprehensive Review on Classification, Energy Management Strategy, and Control Algorithm for Hybrid Electric Vehicles. Energies 2020, 13, 5355. [CrossRef]

5. Sundstrom, O.; Ambuhl, D.; Guzzella, L. On implementation of dynamic programming for optimal control problems with final state constraints. Oil Gas Sci. Technol. Rev. IFP 2010, 65, 91-102. [CrossRef]

6. Sundstrom, O.; Guzzella, L. A generic dynamic programming matlab function. In Proceedings of the IEEE Control Applications, (CCA) \& Intelligent Control, (ISIC), St. Petersburg, Russia, 8-10 July 2009.

7. Wang, R.; Lukic, S.M. Dynamic programming technique in hybrid electric vehicle optimization. In Proceedings of the IEEE International Electric Vehicle Conference, Greenville, SC, USA, 4-8 March 2012.

8. Ozatay, E.; Onori, S.; Wollaeger, J.; Ozguner, U.; Rizzoni, G.; Filev, D.; Michelini, J.; Di Cairano, S. Cloud-based velocity profile optimization for everyday driving: A dynamic-programming-based solution. IEEE Trans. Intell. Transp. Syst. 2014, 15, $2491-2505$. [CrossRef]

9. Chasse, A.; Sciarretta, A. Supervisory Control of Hybrid Powertrains: An Experimental Benchmark of Offline Optimization and Online Energy Management. Control Eng. Pract. 2011, 19, 1253-1265. [CrossRef]

10. Payri, F.; Guardiola, C.; Pla, B.; Blanco-Rodriguez, D. A stochastic method for the energy management in hybrid electric vehicles. Control Eng. Pract. 2014, 29, 257-265. [CrossRef]

11. Onori, S.; Tribioli, L. Adaptive Pontryagin's Minimum Principle supervisory controller design for the plug-in hybrid GM Chevrolet Volt. Appl. Energy 2015, 147, 224-234. [CrossRef]

12. Cervone, D.; Sessa, B.; Arsie, I.; Pianese, C.; Polverino, P. A Comprehensive Hybrid Vehicle Model for Energetic Analysis on Different Powertrain Architectures. SAE Tech. Pap. 2019. [CrossRef]

13. D'Amato, A.; Donatantonio, F.; Arsie, I.; Pianese, C. Enhancing Cruise Controllers through Finite-Horizon Driving Mission Optimization for Passenger Vehicles. SAE Tech. Pap. 2018. [CrossRef]

14. Hegde, B.; Ahmed, Q.; Rizzoni, G. Velocity and energy trajectory prediction of electrified powertrain for look ahead control. Appl. Energy 2020, 279, 115903. [CrossRef]

15. Arsie, I.; Graziosi, M.; Pianese, C.; Rizzo, G.; Sorrentino, M. Control strategy optimizaiton for hybrid electric vehicle via provisional load estimate. Rev. Automot. Eng. 2005, 26, 341-348.

16. Liu, J.; Peng, H. Modeling and Control of a Power-Split Hybrid Vehicle. IEEE Trans. Control Syst. Technol. 2008, 16, $1242-1251$.

17. Bellman, R. Dynamic Programming; Princeton University Press: New York, NY, USA, 1957.

18. Liberzon, D. Calculus of Variations and Optimal Control Theory: A Concise Introduction. Princeton University Press: New York, NY, USA, 2012.

19. Bertsekas, D.P. Dynamic Programming and Optimal Control, Volume I; Athena Scientific: Belmont, MA, USA, 2012. 